\title{
Indications and risk factors for hospitalization in patients with primary Sjögren syndrome: experience from a tertiary center in Turkey
}

\author{
Müçteba Enes Yayla ${ }^{1,2}$. Didem Şahin Eroğlu ${ }^{1}$ Emine Uslu Yurteri ${ }^{1}$. Ayşe Bahar Keleşoğlu Dinçer ${ }^{1} \cdot$ Serdar Sezer $^{1}$. \\ Emine Gözde Aydemir Gülöksüz ${ }^{1}$. Mehmet Levent Yüksel ${ }^{1}$ • Recep Yılmaz ${ }^{1}$ - Aşkın Ateş ${ }^{1}$ Tahsin Murat Turgay ${ }^{1}$. \\ Gülay Kınıklı ${ }^{1}$
}

Received: 11 October 2021 / Revised: 20 December 2021 / Accepted: 31 December 2021 / Published online: 6 January 2022

(c) International League of Associations for Rheumatology (ILAR) 2022

\begin{abstract}
Objective In this study, it was aimed to reveal the hospitalization reasons for patients diagnosed with primary Sjögren syndrome (pSS) and potentially associated factors in a tertiary health center.

Method One hundred and sixty-three pSS patients who regularly attended their follow-ups between January 2010 and May 2021 were included in the study. These patients' reasons for hospitalization, duration of hospitalization, and numbers of presenting to the hospital were recorded. The demographic, clinical and serological characteristics of the hospitalized and non-hospitalized patients were compared.

Results Hospitalization occurred in $22.7 \%$ of the patients, and the total number of hospitalizations was 79 . The hospitalization incidence density rate was 6.21 per 100 patient-years. The most frequently encountered reason for hospitalizations was pSS-related organ involvement (44.3\%). Infections (17.7\%), malignancy (16.5\%), endocrine, and various other reasons were the other indications for hospitalization. While male sex $(p=0.005)$, the presence of extra-glandular involvement $(p<0.001)$, and interstitial lung disease $(p=0.001)$ were more common in the hospitalized patients, anti-nuclear antibody positivity was less frequent $(p=0.032)$. The usage rate of hydroxychloroquine $(p=0.022)$ was lower in the hospitalized patients, whereas the use of glucocorticoids $(p<0.001)$ and azathioprine $(p=0.005)$ was more frequent. The multivariable analyses revealed a relationship between extra-glandular involvement (OR: 4.57 [1.05-19.84], $p=0.043$ ), glucocorticoid use (OR: 3.23 [1.13-9.21], $p=0.028$ ) and hospitalization.

Conclusion pSS-related system involvement and infection accounted for the majority of hospitalizations of the pSS patients. The presence of extra-glandular involvement and glucocorticoid use were found to be associated with hospitalization.

\section{Key Points}

- pSS-related system involvement and infection accounted for the majority of hospitalizations of pSS patients.

- The presence of extra-glandular involvement was found to be associated with hospitalization.
\end{abstract}

Keywords Extra-glandular involvement $\cdot$ Glucocorticoid $\cdot$ Hospitalization $\cdot$ Infection

Müçteba Enes Yayla

enesyayla@hotmail.com

1 Department of Rheumatology, Faculty of Medicine, Ankara University, Ankara, Turkey

2 Clinic of Rheumatology, Ankara Training and Research Hospital, Ankara, Turkey

\section{Introduction}

Primary Sjögren syndrome (pSS) is an autoimmune systemic disease characterized by lymphocytic infiltration of exocrine glands, especially of lacrimal and salivary glands [1]. It is seen more frequently in middle-aged women [2]. Other than the effects on glandular structures, $30-40 \%$ of patients may develop systemic involvements [3]. Pulmonary manifestations such as interstitial lung disease (ILD), pleural effusion and thickening, dermatological findings such as cutaneous vasculitis, subacute cutaneous lupus and erythema 
multiforme, renal involvements such as renal tubular acidosis, glomerulonephritis and interstitial nephritis, peripheral and central nervous system findings such as peripheral, cranial or demyelinating neuropathy and multiple sclerosis-like lesions could be encountered in patients with pSS [4]. The presence of systemic or organ involvement in pSS patients may require immunosuppressive treatment in follow-up [5]. Because of both the effect of immunosuppressive therapy and the clinical burden of the disease, the risk of infection increases in patients, and severe infection can be seen in $10 \%$ of patients $[6,7]$. pSS is also known to be associated with an increased risk of cardiovascular disease and malignancy [6]. Due to such disease-related and comorbidity-originated reasons, hospitalizations are expected situations.

Autoimmune diseases such as systemic lupus erythematosus (SLE) and systemic sclerosis (SSc) have been frequently studied in terms of hospitalization reasons and risk factors, but such studies about pSS are highly limited and very few [8-11]. Hospitalization not only creates an economic burden on the health system, but also leads to a decrease in the quality of life of patients. Knowing the reasons for hospitalization and risk factors in pSS patients may be helpful in reducing the burden on the health system. For this purpose, in this study, we aimed to reveal the hospitalization reasons for patients diagnosed with pSS and potentially associated factors at a tertiary health center.

\section{Materials and methods}

\section{Patient selection}

Three hundred and sixty-seven pSS patients who presented to the outpatient clinic of the Rheumatology Department of the Faculty of Medicine at Ankara University between January 2010 and May 2021 were retrospectively examined. With the aim of selecting patients who attended their follow-up regularly, 163 patients who attended at least one rheumatology visit each year from the day of their first visit were included. All patients had been classified based on the 2016 American College of Rheumatology (ACR)/European League Against Rheumatism (EULAR) pSS Classification [12]. Patients under the age of 18 or those with a concomitant autoimmune connective tissue diseases were excluded.

\section{Definitions}

The patients who were hospitalized at least once for reasons other than elective procedures were defined as the hospitalized patient group. These patients' durations of hospitalization, presence of multiple occasions of hospitalization, status of intensive care unit (ICU) hospitalization, and reasons for hospitalization were recorded. The age that was used in this study was determined as the time between the date of birth and the date of the first hospitalization for the hospitalized group and the time between the date of birth and the date of the last visit for the non-hospitalized group. The duration of disease was defined as the time between the date of the diagnosis and the date of the last visit.

The clinical features of the patients were examined retrospectively from their digital file records. The onset age of disease was described as the time of the first symptom that was considered to be related to pSS. Skin involvement was defined as the presence of Sjögren-related skin findings evaluated by a rheumatologist or dermatologist, such as subacute cutaneous lupus (papulosquamous lesions and annular lesions that develop in sun-exposed areas, including the upper back, shoulders, extensor arms, neck, and upper torso) or cutaneous vasculitis. Pulmonary involvement was determined as the presence of ILD, pulmonary arterial hypertension (PAH), pleural effusion, or pleural thickening. ILD was determined as the presence of ground glass opacities, reticulation, tractional bronchiectasis, or honeycombing in high resolution computed tomography (HRCT). The presence of pleural effusion or thickening was evaluated with HRCT. PAH was defined as the mean systolic pulmonary arterial pressure $\geq 25 \mathrm{mmHg}$ and pulmonary capillary wedge pressure $<15 \mathrm{mmHg}$ following right cardiac catheterization. Urinary system involvement was defined as the presence of renal tubular acidosis, glomerulonephritis, interstitial nephritis, or interstitial cystitis. Renal tubular acidosis was defined as hyperchloremia, low bicarbonate level, and presence of acidosis as a result of blood gas analysis. The diagnosis of glomerulonephritis, interstitial nephritis, or interstitial cystitis was confirmed pathologically. Peripheral nerve involvement was defined as the presence of sensory or motor neuropathy, demyelinating neuropathy, or entrapment neuropathy confirmed with electromyoneurography or imaging methods. Central nervous system involvement was accepted as the presence of seizures or cranial nerve involvement, optic neuritis, multiple sclerosis-like involvement, cerebral vasculitis, and transverse myelitis confirmed with magnetic resonance imaging. Other non-pSS causes (infectious, toxic, and other) were excluded by neurological examination. Hematological involvement was described as the presence of leukopenia, lymphopenia, and thrombocytopenia considered to be of autoimmune origin or autoimmune hemolytic anemia. The presence of skin, pulmonary, urinary system, peripheral nervous system, central nervous system, or hematological involvement in the patients was defined as the presence of extra-glandular involvement. The comorbidity statuses of the patients were evaluated with the Charlson Comorbidity Index [13]. From the patient files, rheumatoid factor (RF) and immunoglobulin (Ig) G levels, anti-nuclear antibody (ANA), Ro-52, and SS-B values were recorded. 


\section{Statistical analysis}

The data were analyzed using the SPSS version 21 software (SPSS, Chicago, USA). The categorical data are presented using frequencies and percentages. The quantitative data are presented as medians and interquartile ranges (IQR) (nonnormally distributed). In the comparison of the categorical data of the hospitalized and non-hospitalized patients, Chi-Square test or Fisher's exact test was used based on suitability. Mann-Whitney $U$ test was used to compare the quantitative data. Multivariable analysis was performed by logistic regression analysis, which modeled the independent predictors of hospitalization, by using possible factors identified in the univariate analyses (variables with a $p$ value $<0.25$ in the univariate analyses were subsequently entered into the final multivariable model). In this analysis, the non-hospitalized group of patients was taken as the reference group. The odds ratios (OR) calculated as a result of these analyses were defined using $95 \%$ confidence intervals. Hosmer-Lemeshow test was used to assess goodness-of-fit. A $p<0.05$ was considered statistically significant.

\section{Results}

Hospitalization occurred in 22.7\% (37/163) of the patients, and the total number of hospitalizations was 79 . The incidence density rate per 100 patient-years was 6.21 . The median duration of hospital stay was 8 (IQR 10) days. There was a history of recurrent hospitalization among $43.2 \%$ (16/37) of the hospitalized patients. The most frequently encountered reason for hospitalizations was pSS-related system/tissue involvement (44.3\%). Infections (17.7\%), malignancy $(16.5 \%)$, endocrine-metabolic $(3.5 \%)$, and various other reasons $(17.7 \%)$ were the other indications for hospitalization (Table 1).

In 8 of the patients, the total number of ICU hospitalizations was 10 . The reasons for ICU hospitalizations were $\operatorname{ILD}(n=2)$, bacterial pneumonia $(n=2)$, endometrial cancer $(n=2)$, colon cancer $(n=2)$, coronavirus disease-19 (COVID-19) pneumonia $(n=1)$, and PAH $(n=1)$. The median duration of ICU stay was 3.5 (IQR 16.75) days.

Of the hospitalized patients, $18.9 \%$ (7/37) died. The causes of death of these patients were bacterial pneumonia
Table 1 Reasons for hospitalization

\begin{tabular}{|c|c|}
\hline \multicolumn{2}{|l|}{ Primary Sjögren associated causes $(N=35,44.3 \%)$} \\
\hline & $\mathrm{ILD}, N=12$ \\
\hline & Urinary system involvement, $N=8$ \\
\hline & CNS Involvement, $N=4$ \\
\hline & Eye complication, $N=4$ \\
\hline & $\mathrm{PAH}, N=3$ \\
\hline & Arthritis, $N=3$ \\
\hline & Pleural effusion, $N=1$ \\
\hline \multicolumn{2}{|l|}{ Infection $(N=14,17.7 \%)$} \\
\hline & Bacterial pneumonia, $N=7$ \\
\hline & COVID-19 pneumonia, $N=3$ \\
\hline & GIS infection, $N=2$ \\
\hline & Urinary tract infection, $N=1$ \\
\hline & Invasive candidiasis, $N=1$ \\
\hline \multicolumn{2}{|l|}{ Malignancy $(N=13,16.5 \%)$} \\
\hline & Endometrium CA, $N=10$ \\
\hline & Colon CA, $N=2$ \\
\hline & Mycosis fungoides, $N=1$ \\
\hline \multicolumn{2}{|l|}{ Endocrine metabolism $(N=3,3.8 \%)$} \\
\hline & Severe hypercalcemia, $N=2$ \\
\hline & Severe hypocalcemia, $N=1$ \\
\hline \multicolumn{2}{|l|}{ Miscellaneous $(N=14,17.7 \%)$} \\
\hline & Orthopedic problems, $N=4$ \\
\hline & Dermatological problems, $N=4$ \\
\hline & GIS abnormalities, $N=3$ \\
\hline & Major depression, $N=1$ \\
\hline & Unknown origin inflammation, $N=1$ \\
\hline & Submandibular mass, $N=1$ \\
\hline
\end{tabular}


$(n=2)$, ILD $(n=2)$, PAH $(n=1)$, COVID-19 pneumonia

$(n=1)$, and endometrial cancer $(n=1)$.

While male sex $(p=0.005)$, the presence of extra-glandular involvement $(p<0.001)$, and ILD $(p=0.001)$ were more common in the hospitalized patients, ANA positivity was less frequent $(p=0.032)$. The usage rate of hydroxychloroquine $(p=0.022)$ was lower in the hospitalized patients, whereas the use of glucocorticoids $(p<0.001)$ and azathioprine $(p=0.005)$ was more frequent (Table 2$)$.

The results of the univariate analyses of potentially related factors for hospitalization are presented in Table 3, and the results of multivariable logistic regression analyses are shown in Table 4. In the multivariable analyses, the variables that were found to be significantly associated with hospitalization were extra-glandular involvement (OR: 4.57 [1.05-19.84], $p=0.043$ ) and glucocorticoid use (OR: 3.23 [1.13-9.21], $p=0.028$ ) in Model 1, glucocorticoid use $(\mathrm{OR}=2.92$ [1.06-8.06], $p=0.039)$ in Model 2, and extraglandular involvement (OR: 3.68 [1.43-9.42], $p=0.007$ ) and glucocorticoid use (OR: 2.85 [1.11-7.37], $p=0.030$ ) in Model 3.

\section{Discussion}

Hospitalization constitutes a significant reason for health expenses. Therefore, the reasons for hospitalization, especially in connective tissue diseases, have been frequently studied [14-18]. However, there are very few studies conducted on this topic with pSS patients $[10,11]$. In this study, we aimed to reveal the hospitalization reasons for pSS patients at a tertiary health institution. It is known that hospitalization is more frequent in pSS patients compared to the healthy population [11]. Previous studies have reported the hospitalization incidence density rates of pSS patients to be in the range of 6.49-24.2 per 100 patient-years [10, 11]. In our study, we found the incidence density rate as 6.21 per 100 patient-years in pSS patients. It was seen that
Table 2 Comparison of hospitalized and nonhospitalized patients

\begin{tabular}{|c|c|c|c|c|}
\hline & $\begin{array}{l}\text { All pSS patients } \\
N=163\end{array}$ & $\begin{array}{l}\text { Hospitalized } \\
\text { pSS patients } \\
N=37\end{array}$ & $\begin{array}{l}\text { Non-hospitalized } \\
\text { pSS patients } \\
N=126\end{array}$ & $p$ \\
\hline Sex, female* & $154(94.5)$ & $31(83.8)$ & $123(97.6)$ & 0.005 \\
\hline Age at diagnosis, year $\dagger$ & $55.14[17.42]$ & $57.18[16.6]$ & $53.48[17.42]$ & 0.143 \\
\hline Age, year $\dagger$ & $61.76[14.86]$ & $61.02[14.63]$ & $62.16[15.37]$ & 0.452 \\
\hline Disease duration, year $\dagger$ & $7.62[5.28]$ & $6.96[5.16]$ & $7.81[5.28]$ & 0.325 \\
\hline \multicolumn{5}{|l|}{ Clinical manifestations } \\
\hline Extra-glandular involvement* & $62(38)$ & $25(67.6)$ & $38(30.2)$ & $<0.001$ \\
\hline Arthritis* & $28(17.2)$ & $6(16.2)$ & $22(17.5)$ & 0.860 \\
\hline Skin involvement* & $8(4.9)$ & $4(10)$ & $4(3.2)$ & 0.079 \\
\hline Pulmonary involvement* & $27(16.6)$ & $13(35.1)$ & $14(11.1)$ & 0.001 \\
\hline Urinary system involvement* & $3(1.8)$ & $3(8.1)$ & 0 & 0.011 \\
\hline PNS involvement* & $8(4.9)$ & $1(2.7)$ & $7(5.6)$ & 0.684 \\
\hline CNS involvement* & $8(4.9)$ & $4(10.8)$ & $4(3.2)$ & 0.079 \\
\hline Hematological involvement* & $31(19)$ & $10(27)$ & $21(16.7)$ & 0.158 \\
\hline Charlson's comorbidity score $\dagger$ & $3[2]$ & $3[2]$ & $3[2]$ & 0.974 \\
\hline \multicolumn{5}{|l|}{ Serological features* } \\
\hline ANA positivity & $117 / 160(73.1)$ & $22 / 37(59.5)$ & $95 / 123(77.2)$ & 0.032 \\
\hline Ro-52positivity & $81 / 147(55.1)$ & $20 / 33(60.6)$ & $61 / 114(53.5)$ & 0.470 \\
\hline SS-B positivity & $37 / 147(25.2)$ & $11 / 33(33.3)$ & $26 / 114(22.8)$ & 0.220 \\
\hline RF positivity & $36 / 160(22.5)$ & $9 / 36(25)$ & $27 / 124(21.8)$ & 0.683 \\
\hline Increased IgG & $33 / 110(30)$ & $10 / 29(34.5)$ & 23/81 (28.4) & 0.539 \\
\hline \multicolumn{5}{|l|}{ Medications* } \\
\hline Hydroxychloroquine & $139 / 159(87.4)$ & 28/37 (75.7) & $111 / 122(91)$ & 0.022 \\
\hline Glucocorticoid & $39 / 159(24.5)$ & $18 / 37(48.6)$ & $21 / 122(17.2)$ & $<0.001$ \\
\hline Azathioprine & $17 / 159(10.7)$ & $9 / 37(24.3)$ & $8 / 122(6.6)$ & 0.005 \\
\hline Methotrexate & $12 / 159(7.5)$ & 4/37 (10.8) & $8 / 122(6.6)$ & 0.476 \\
\hline Mycophenolate mofetil & $5 / 159(3.1)$ & $2 / 37(5.4)$ & $3 / 122(2.5)$ & 0.330 \\
\hline Rituximab & $2 / 159(1.3)$ & $1 / 37(2.7)$ & $1 / 122(0.8)$ & 0.412 \\
\hline Cyclophosphamide & $2 / 159(1.3)$ & $1 / 37(2.7)$ & $1 / 122(0.8)$ & 0.412 \\
\hline
\end{tabular}

$P$ values that are considered statistically significant are written in bold 
Table 3 Univariate analysis of possible factors associated with hospitalization

\begin{tabular}{llr}
\hline & OR 95\% CI & $p$ \\
\hline Male & $7.93[1.88-33.52]$ & $\mathbf{0 . 0 0 5}$ \\
Age at diagnosis, year & $1.02[0.99-1.05]$ & 0.213 \\
Disease duration, year & $0.98[0.90-1.07]$ & 0.648 \\
Extra-glandular involvement & $4.82[2.20-10.59]$ & $<\mathbf{0 . 0 0 1}$ \\
Arthritis & $0.91[0.34-2.46]$ & 0.860 \\
Skin involvement & $3.70[0.88-15.58]$ & 0.075 \\
Pulmonary involvement & $4.33[1.81-10.39]$ & $\mathbf{0 . 0 0 1}$ \\
PNS involvement & $0.47[0.06-3.97]$ & 0.490 \\
CNS involvement & $3.70[0.88-15.58]$ & 0.075 \\
Hematological involvement & $1.85[0.78-4.39]$ & 0.162 \\
Charlson's comorbidity score & $1.02[0.76-1.37]$ & 0.877 \\
ANA positivity & $0.43[0.20-0.94]$ & $\mathbf{0 . 0 3 5}$ \\
Ro-52 positivity & $1.34[0.61-2.94]$ & 0.471 \\
Hydroxychloroquine & $0.31[0.12-0.82]$ & $\mathbf{0 . 0 1 8}$ \\
Glucocorticoids & $4.56[2.05-10.12]$ & $<\mathbf{0 . 0 0 1}$ \\
Azathioprine & $4.58[1.62-12.94]$ & $\mathbf{0 . 0 0 4}$ \\
\hline
\end{tabular}

$P$ values that are considered statistically significant are written in bold

this hospitalization rate was lower than those in cases of other autoimmune connective tissue diseases such as SSc and SLE [19]. The risk of infection may be increased in SSc and SLE patients due to the higher frequency of severe organ involvement and the need for intensive immunosuppressive therapy, and this may explain why hospitalizations are more frequent in these patients.

In our study, pSS-related system/tissue involvement, infection and malignancy were the most frequently encountered reasons for hospitalization (Table 1). A single-center study conducted in Mexico showed Sjögren disease activation, infection, and malignancy as the most prevalent causes of hospitalization in pSS patients [10]. In a retrospective population-based study in the USA, cardiovascular system, connective tissue and musculoskeletal system disorders, respiratory system disorders, and injuries were observed to be among the most prevalent reasons for hospitalization [11]. In studies conducted on other rheumatic diseases, it has been seen in similarity to pSS patients that disease activation and infection were the main reasons for hospitalization. The most common causes of hospitalization in SLE patients are SLE flares and organ involvement, infection, cardiovascular system diseases, and pregnancy-related conditions $[9,15$, $16,20,21]$, whereas those in SSc patients are SSc-related causes (PAH, ILD, digital ulcer, renal crisis), infection, and heart failure [17, 18, 22].

Studies investigating hospitalization reasons in pSS patients have not reported whether or not there is a relationship between sex and hospitalization frequency $[10,11]$. Some studies examining the effects of sex on hospitalization status in SLE could not show a relationship between hospitalization and sex $[14,15]$. In our study, we found that male sex was more common in the hospitalized patients, and in the univariate analyses, we found that male sex was significantly associated with hospitalization, but this statistical significance was not observed in the multivariable analyses (Tables 2, 3, and 4). Studies examining the characteristics of male pSS patients have shown that the frequency of pulmonary involvement and lymphoma is higher in males [23, 24]. Hospitalizations may be more frequent in male pSS patients because of these extra-glandular involvements, and there is a need for comprehensive cohort studies on this topic.

To the best of our knowledge, there is only one study that investigated the relationship between hospitalization and clinical findings in pSS patients. The study proposed the idea that there may be a relationship between hospitalization and the presence of vasculitis, short disease duration, glomerulonephritis, neurological involvement, hepatic involvement, and hyper viscosity [10]. It was also reported that

Table 4 Multivariable logistic regression analysis of possible factors associated with hospitalization

\begin{tabular}{|c|c|c|c|c|c|c|}
\hline & $\begin{array}{l}\text { Model 1* } \\
\text { OR 95\% CI }\end{array}$ & $p$ & $\begin{array}{l}\text { Model 2** } \\
\text { OR 95\% CI }\end{array}$ & $p$ & $\begin{array}{l}\text { Model 3*** } \\
\text { OR 95\% CI }\end{array}$ & $p$ \\
\hline Male & $5.33[0.94-30.16]$ & 0.058 & $4.74[0.91-24.86]$ & 0.066 & $4.74[0.86-6.03]$ & 0.073 \\
\hline Age of diagnosis & $1.01[0.97-1.05]$ & 0.740 & $1.01[0.97-1.04]$ & 0.764 & $1.00[0.96-1.04]$ & 0.975 \\
\hline Extra-glandular involvement & $4.57[1.05-19.84]$ & 0.043 & - & - & $3.68[1.43-9.42]$ & 0.007 \\
\hline Skin involvement & $2.58[0.41-16.27]$ & 0.312 & $5.20[0.86-31.60]$ & 0.073 & - & - \\
\hline Pulmonary involvement & $0.61[0.12-3.16]$ & 0.558 & $1.75[0.47-6.51]$ & 0.401 & - & - \\
\hline CNS involvement & $0.71[0.08-6.01]$ & 0.751 & 1.98 [0.30-13.09] & 0.480 & - & - \\
\hline Hematological involvement & $0.76[0.21-2.67]$ & 0.666 & $1.61[0.55-4.72]$ & 0.388 & - & - \\
\hline ANA positivity & $0.47[0.17-1.25]$ & 0.128 & $0.49[0.19-1.27]$ & 0.143 & $0.47[0.18-1.21]$ & 0.116 \\
\hline Hydroxychloroquine & $0.34[0.10-1.17]$ & 0.088 & $0.38[0.11-1.26]$ & 0.112 & $0.34[0.10-1.16]$ & 0.084 \\
\hline Glucocorticoids & $3.23[1.13-9.21]$ & 0.028 & $2.92[1.06-8.06]$ & 0.039 & 2.85 [1.11-7.37] & $\mathbf{0 . 0 3 0}$ \\
\hline Azathioprine & $1.52[0.37-6.17]$ & 0.557 & $1.51[0.38-5.94]$ & 0.556 & $1.34[0.36-5.00]$ & 0.660 \\
\hline
\end{tabular}

$P$ values that are considered statistically significant are written in bold 
hospitalization was encountered among pSS patients with renal involvement [25]. In our study, the prevalence of extraglandular involvement, pulmonary involvement, or urinary system involvement was higher in the patients who were hospitalized (Table 2). The multivariable analyses in this study showed a statistically significant relationship between the presence of extra-glandular involvement and hospitalization (Table 4). In patients with extra-glandular involvement, the disease may be more severe, and immunosuppressive treatment may be required. Both the severity of the disease and an increase in the risk of infection after immunosuppressive treatment appear to be raising the prevalence of hospitalization in these patients.

It has been shown that ANA-positive pSS patients are diagnosed younger and female sex predominates in these patients [26]. There are also other studies reporting no significant relationship of extra-glandular findings and disease activity with ANA positivity [26, 27]. We found ANA positivity more frequently in the non-hospitalized patients in our study, but we did not find a statistically significant relationship between hospitalization and the presence of ANA positivity in the multivariable analyses. It is known that there is a link between systemic involvement and anti-Ro52/ SS-A in pSS patients [27]. However, we could not establish a link similar to those in other studies between the presence of anti-Ro52 and hospitalization [10].

It was reported that hydroxychloroquine reduces hospitalization rates among SLE patients [15]. It is thought that the drug shows this effect by reducing disease exacerbation in these patients $[28,29]$. It has been suggested that the use of hydroxychloroquine in pSS patients may also have a protective role against hospitalization [10]. In our study, the rate of hydroxychloroquine usage was lower in the hospitalized patients. Hydroxychloroquine might be preventing hospitalization. Nevertheless, factors such as the possibility that hydroxychloroquine were used more frequently in the patients with mild disease activity and that hydroxychloroquine was not preferred for use in those needing immunosuppressive treatment might have created a bias in this result.

In the presence of systemic involvement in pSS patients, it may be necessary to use glucocorticoids and immunosuppressive treatment. The intensity and duration of immunosuppressive therapy may increase, especially in severe disease involvement [5]. It is also a known fact that the risk of infection increases with immunosuppressive treatment. In our patients, we showed that the most significant factors in hospitalizations were system/tissue involvement and infection (Table 1). Therefore, we may state that the frequent use of glucocorticoids and azathioprine in hospitalized patients is an expected result.

This study has some limitations such as having a singlecenter and retrospective design, the absence of disease activation scores and minor salivary gland biopsy focus scores, the lack of data on cryoglobulin, and complement levels, which are some poor prognostic markers. In our study, in which patients who attended their follow-ups regularly were included, the main limitation was that we included no data on patients who were in a severe condition and were hospitalized or died at another center. Moreover, a strength of our study that needs to be stated is that it is one of very few studies where the hospitalization reasons and hospitalizationrelated factors of pSS patients have been studied.

In conclusion, in this study, pSS-related system/tissue involvement and infection accounted for most of the hospitalizations among the pSS patients. The presence of extraglandular involvement and the use of glucocorticoids were associated with hospitalization.

\section{Declarations}

\section{Disclosures None.}

$C N S$ central nervous system, GIS gastrointestinal system, ILD interstitial lung disease, $N$ number of patients, $P A H$ pulmonary arterial hypertension.

$A N A$ anti-nuclear antibody, $C N S$ central nervous system, $N$ number of patients, $P N S$ peripheral nervous system, $R F$ rheumatoid factor.*Data presented as numbers and percentages. $\dagger$ Data presented as medians and interquartile range.

$A N A$ anti-nuclear antibody, $C I$ confidence interval, $C N S$ central nervous system, $O R$ odds ratio, $P N S$ peripheral nervous system.

$A N A$ anti-nuclear antibody, $C I$ confidence interval, $C N S$ central nervous system, $O R$ odds ratio.*Hosmer and Lemeshow test $p=0.431$; ChiSquare: 8.203.**Hosmer and Lemeshow test $p=0.635$; Chi-Square: 6.111 ; since there was a high correlation between extra-glandular involvement and skin, pulmonary, CNS, and hematological involvement, extra-glandular involvement was excluded, and modeling was performed in Model 2.***Hosmer and Lemeshow test $p=0.451$; Chi-Square: 7.819; since there was a high correlation between extraglandular involvement and skin, pulmonary, CNS and hematological involvement, modeling was performed by excluding skin, pulmonary, CNS and hematological involvement in Model 3.

\section{References}

1. Ramos-Casals M, Font J (2005) Primary Sjogren's syndrome: current and emergent aetiopathogenic concepts. Rheumatology (Oxford) 44:1354-1367

2. Kassan SS, Moutsopoulos HM (2004) Clinical manifestations and early diagnosis of Sjögren syndrome. Arch Intern Med $164: 1275-1284$

3. Mariette X, Criswell LA (2018) Primary Sjögren's syndrome. N Engl J Med 378:931-939

4. Seror R, Ravaud P, Bowman SJ, Baron G, Tzioufas A, Theander E, Gottenberg J-E, Bootsma H, Mariette X, Vitali C (2010) EULAR Sjögren's syndrome disease activity index: development of a consensus systemic disease activity index for primary Sjögren's syndrome. Ann Rheum Dis 69:1103-1109

5. Ramos-Casals M, Brito-Zerón P, Bombardieri S, Bootsma H, De Vita S, Dörner T, Fisher BA, Gottenberg J-E, HernandezMolina G, Kocher A (2020) EULAR recommendations for the 
management of Sjögren's syndrome with topical and systemic therapies. Ann Rheum Dis 79:3-18

6. Rúa-Figueroa I, Fernández Castro M, Andreu JL, Sanchez-Piedra C, Martínez-Taboada V, Olivé A, López-Longo J, Rosas J, Galindo M, Calvo-Alén J (2017) Comorbidities in patients with primary Sjögren's syndrome and systemic lupus erythematosus: a comparative registries-based study. Arthritis Care Res 69:38-45

7. Singh JA, Cleveland JD (2020) Serious infections in Sjögren's syndrome patients: a national US study. Clin Exp Rheumatol 38:S47-S52

8. Chung L, Krishnan E, Chakravarty E (2007) Hospitalizations and mortality in systemic sclerosis: results from the Nationwide Inpatient Sample. Rheumatology (Oxford) 46:1808-1813

9. Manadan AM, Kambhatla S, Gauto-Mariotti E, Okoli C, Block JA (2020) Reasons for hospitalization and in-hospital mortality in adult systemic lupus erythematosus. ACR open rheumatology 2:683-689

10. Atisha-Fregoso Y, Rivera-Vicencio Y, Baños-Pelaez M, Hernández-Molina G (2015) Main causes and risk factors for hospitalisation in patients with primary Sjögren's syndrome. Clin Exp Rheumatol 33:721-725

11. Maciel G, Servioli L, Nannini C, Berti A, Crowson CS, Achenbach SJ, Matteson EL, Cornec D (2018) Hospitalisation rates among patients with primary Sjögren's syndrome: a populationbased study, 1995-2016. RMD open 4:e000575.

12. Shiboski CH, Shiboski SC, Seror R, Criswell LA, Labetoulle M, Lietman TM, Rasmussen A, Scofield H, Vitali C, Bowman SJ (2017) 2016 American College of Rheumatology/European League Against Rheumatism classification criteria for primary Sjögren's syndrome: a consensus and data-driven methodology involving three international patient cohorts. Arthritis Rheumatol 69:35-45

13. Charlson ME, Pompei P, Ales KL, MacKenzie CR (1987) A new method of classifying prognostic comorbidity in longitudinal studies: development and validation. J Chronic Dis 40:373-383

14. Lee J, Park D, Kang J, Choi S, Yim Y, Kim J, Lee K, Wen L, Kim T, Park Y (2016) The rate of and risk factors for frequent hospitalization in systemic lupus erythematosus: results from the Korean lupus network registry. Lupus 25:1412-1419

15. Liang H, Pan HF, Tao JH, Ye DQ (2019) Causes and factors associated with frequent hospitalization in Chinese patients with systemic lupus erythematosus: an ambispective cohort study. Med Sci Monit 25:8061

16. Anandarajah A, Luc M, Ritchlin C (2017) Hospitalization of patients with systemic lupus erythematosus is a major cause of direct and indirect healthcare costs. Lupus 26:756-761

17. Netwijitpan S, Foocharoen C, Mahakkanukrauh A, Suwannaroj S, Nanagara R (2013) Indications for hospitalization and in-hospital mortality in Thai systemic sclerosis. Clin Rheumatol 32:361-367

18. Coffey CM, Sandhu AS, Crowson CS, Achenbach SJ, Matteson EL, Osborn TG, Warrington KJ, Makol A (2021) Hospitalization rates are highest in the first 5 years of systemic sclerosis: results from a population-based cohort (1980-2016). J Rheumatol 48:877-882

19. Foocharoen C, Thavornpitak Y, Mahakkanukrauh A, Suwannaroj S, Nanagara R (2013) Admission rate and characteristics of hospitalized systemic connective tissue disorders: analysis from a nationwide Thailand healthcare database. Int J Rheum Dis 16:41-46

20. Edwards C, Lian T, Badsha H, Teh C, Arden N, Chng H (2003) Hospitalization of individuals with systemic lupus erythematosus: characteristics and predictors of outcome. Lupus 12:672-676

21. Lee J, Dhillon N, Pope J (2013) All-cause hospitalizations in systemic lupus erythematosus from a large Canadian referral centre. Rheumatology (Oxford) 52:905-909

22. Shenavandeh S, Naseri R (2017) Assessment of hospitalization and mortality of scleroderma in-patients: a thirteen-year study. Reumatologia 55:163

23. Park Y, Lee J, Park SH, Kwok SK (2020) Male patients with primary Sjögren's syndrome: a distinct clinical subgroup? Int J Rheum Dis 23:1388-1395

24. Chatzis L, Pezoulas VC, Ferro F, Gandolfo S, Donati V, Binutti M, Callegher SZ, Venetsanopoulou A, Zampeli E, Mavrommati M (2020) Sjögren's syndrome: the clinical spectrum of male patients. J Clin Med 9:2620

25. Narvaez J, Sánchez-Piedra C, Fernández-Castro M, MartínezTaboada V, Olivé A, Rosas J, García-Vadillo JA, Júdez E, RuizLucea E, Romani L (2020) Clinically significant renal involvement in primary Sjögren's syndrome is associated with important morbidity: data from the Spanish Sjögrenser cohort. Clin Exp Rheumatol 38:S116-S124

26. Huo AP, Lin KC, Chou CT (2010) Predictive and prognostic value of antinuclear antibodies and rheumatoid factor in primary Sjogren's syndrome. Int J Rheum Dis 13:39-47

27. Ramos-Casals M, Brito-Zerón P, Solans R, Camps M-T, Casanovas A, Sopeña B, Díaz-López B, Rascón F-J, Qanneta R, Fraile G (2014) Systemic involvement in primary Sjögren's syndrome evaluated by the EULAR-SS disease activity index: analysis of 921 Spanish patients (GEAS-SS Registry). Rheumatology (Oxford) 53:321-331

28. Alarcón GS, McGwin G, Bertoli AM, Fessler BJ, Calvo-Alén J, Bastian HM, Vilá LM, Reveille JD (2007) Effect of hydroxychloroquine on the survival of patients with systemic lupus erythematosus: data from LUMINA, a multiethnic US cohort (LUMINA L). Ann Rheum Dis 66:1168-1172

29. Akhavan PS, Su J, Lou W, Gladman DD, Urowitz MB, Fortin PR (2013) The early protective effect of hydroxychloroquine on the risk of cumulative damage in patients with systemic lupus erythematosus. J Rheumatol 40:831-841

Publisher's note Springer Nature remains neutral with regard to jurisdictional claims in published maps and institutional affiliations. 